\title{
Knockdown of cytokeratin 8 overcomes chemoresistance of chordoma cells by aggravating endoplasmic reticulum stress through PERK/eIF2a arm of unfolded protein response and blocking autophagy
}

\author{
Di Wang ${ }^{1}$, Peiran Zhang ${ }^{2}$, Xiaolong Xu' ${ }^{1}$, Jianhui Wang ${ }^{1}$, Dong Wang ${ }^{1}$, Pandi Peng ${ }^{1,3}$, Chao Zheng ${ }^{1}$, Qing-Jun Meng ${ }^{4}$, \\ Liu Yang ${ }^{1,3}$ and Zhuojing Luo ${ }^{1,3}$
}

\begin{abstract}
Chordoma is a malignant primary osseous spinal tumor with pronounced chemoresistance. However, the mechanisms of how chordoma cells develop chemoresistance are still not fully understood. Cytokeratin 8 (KRT8) is a molecular marker of notochordal cells, from which chordoma cells were believed to be originated. In this study, we showed that either doxorubicin or irinotecan promoted KRT8 expression in both CM319 and UCH1 cell lines, accompanied by an increased unfolded protein response and autophagy activity. Then, siRNA-mediated knockdown of KRT8 chemosensitized chordoma cells in vitro. Mechanistic studies showed that knockdown of KRT8 followed by chemotherapy aggravated endoplasmic reticulum stress through PERK/elF2a arm of unfolded protein response and blocked late-stage autophagy. Moreover, suppression of the PERK/elF2a arm of unfolded protein response using PERK inhibitor GSK2606414 partially rescued the apoptotic chordoma cells but did not reverse the blockage of the autophagy flux. Finally, tumor xenograft model further confirmed the chemosensitizing effects of siKRT8. This study represents the first systematic investigation into the role of KRT8 in chemoresistance of chordoma and our results highlight a possible strategy of targeting KRT8 to overcome chordoma chemoresistance.
\end{abstract}

\section{Introduction}

Chordoma is a malignant primary osseous spinal tumor (POST) with aggressive local expansion and pronounced chemoresistance $^{1-3}$. It can occur anywhere along the spine, from the skull base to the sacrum, and account for about $20 \%$ of POSTs. To date, surgery and adjuvant

\footnotetext{
Correspondence: Liu Yang (yangliu@fmmu.edu.cn) or Zhuojing Luo (zjluo@fmmu.edu.cn)

'Institute of Orthopedic Surgery, Xijing Hospital, Fourth Military Medical University, Xi'an 710032, China

${ }^{2}$ Department of Biochemistry and Molecular Biology, Fourth Military Medical University, Xi'an 710032, China

Full list of author information is available at the end of the article.

These authors contributed equally: Di Wang, Peiran Zhang, and Xiaolong Xu Edited by G.M. Fimia
}

radiotherapy remain the foundation of the treatment ${ }^{4,5}$. Most median survivals reported are about 5 years in duration due to its high recurrence rates after en bloc resection. Moreover, conventional chordoma were badly insensitive to cytotoxic chemotherapy, which was to be the standard treatment option for metastatic sarcoma ${ }^{6-9}$. Thus, understanding the underlying reasons and molecular mechanisms of chemoresistance of chordoma would be of great help to improve the prognosis of chordoma patients.

Cytokeratins are a subfamily of intermediate filament proteins and are characterized by a remarkable biochemical diversity ${ }^{10}$. Keratin, type II cytoskeletal 8 , also known as keratin 8 , cytokeratin $8,(K R T 8, C K 8, K 8)$, a

\section{(c) The Author(s) 2019}

(c) (i) Open Access This article is licensed under a Creative Commons Attribution 4.0 International License, which permits use, sharing, adaptation, distribution and reproduction cc. in any medium or format, as long as you give appropriate credit to the original author(s) and the source, provide a link to the Creative Commons license, and indicate if changes were made. The images or other third party material in this article are included in the article's Creative Commons license, unless indicated otherwise in a credit line to the material. If material is not included in the article's Creative Commons license and your intended use is not permitted by statutory regulation or exceeds the permitted use, you will need to obtain permission directly from the copyright holder. To view a copy of this license, visit http://creativecommons.org/licenses/by/4.0/. 
well-known epithelial marker protein, is a molecular marker of notochordal cells, from which chordoma cells were believed to be originated ${ }^{11-13}$. Previous studies have revealed that KRT8 not only contributes to responding mechanical stress, but also has many significant nonmechanical functions such as signal transduction, stem cell differentiation, and cell protection ${ }^{10,14-22}$. Yet, a role of KRT8 in chemoresistance has not been documented.

Endoplasmic reticulum (ER), a network of membranous tubules within the cytoplasm of all eukaryotic cell, plays a pivotal role in protein folding, lipid biosynthesis, calcium signaling, and drug detoxification. The accumulation or aggregation of unfolded/misfolded proteins inside the ER induces a cellular condition known as the ER stress and then triggers a set of intracellular signaling pathways collectively referred to as the unfolded protein response (UPR), to transcriptionally and translationally improve ER protein-folding capacity. Three classical arms of UPR are regulated by three ER membrane-embedded sensors: (1) double-stranded RNA-activated protein kinase-like ER kinase (PERK), (2) inositol-requiring enzyme 1 (IRE1), and (3) activating transcription factor 6 (ATF6) ${ }^{23-26}$. Many drug-resistant tumor cells can utilize diverse strategies that enable them to survive the chemotherapy ${ }^{27}$. Drugs disturbing the protein-folding capacity of the ER can provoke ER stress and subsequently induce UPR, endowing malignant cells with greater tumorigenic, metastatic, and drug-resistant capacity ${ }^{28-30}$.

Macroautophagy (hereafter autophagy) serves as an evolutionarily conserved catabolic and quality-control pathway across all eukaryotes ${ }^{31,32}$. The formation of the phagophore, the initial sequestering compartment, which expands into an autophagosome, marks the initiation of the autophagy ${ }^{33}$. Then, autophagosome fuses with lysosomes followed by degradation of the contents, allowing complete flux through the autophagy pathway. In general, autophagy promotes cell survival in response to starvation or other types of cellular stress. Enhanced autophagic responses can support cancer cell survival, proliferation, and growth in adverse microenvironmental conditions, such as the presence of chemotherapy, thereby contributing to drug resistance ${ }^{34-37}$. Unfortunately, the mechanisms of how chordoma cells develop chemoresistance are complicated and still remain elusive.

In the present study, we found the expression of KRT8 was upregulated in two chordoma cell lines, CM319 and $\mathrm{UCH1}$, after the treatment with doxorubicin (Doxo) or irinotecan (Irino). Therefore, we hypothesized that KRT8 plays a potential role in chemoresistance of chordoma cells. We then used small interfering (siRNA) to knock down the KRT8 expression in chordoma cells followed by chemotherapy both in vitro and in vivo, and the results showed that knockdown of KRT8 overcomes chemoresistance of the chordoma cells through aggravating ER stress, through the PERK/eIF2 $\alpha$ arm of UPR and thereby blocking autophagy. The data from this study are the first to provide compelling evidence that upregulation of KRT8 is one of the mechanism responsible for the chemoresistance of chordoma cells and provided a potential therapeutic approach to overcome chemoresistance of chordoma cells.

\section{Results \\ Doxorubicin or irinotecan significantly promoted KRT8 expression in chordoma cells in vitro}

We first investigated the effect of Doxo $(0.5 \mu \mathrm{M})$ and Irino $(50 \mu \mathrm{M})$ on KRT8 expression of CM319 and $\mathrm{UCH} 1$ chordoma cells, and found that chemotherapy significantly promoted the expression of KRT8 in CM319 and $\mathrm{UCH} 1$ cells in a time-dependent manner, as shown by the quantitative reverse-transcriptase PCR (qRT-PCR) analysis (Fig. 1a). In addition, consistent with qRT-PCR results, the KRT8 expression was significantly increased at $24 \mathrm{~h}$ in both CM319 and UCH1 cell lines as shown by the western blotting analysis (Fig. 1b). To further investigate the reorganization of KRT8 after chemotherapy, we used immunocytochemistry analysis and the results showed that the KRT8 expression was promoted throughout the cell in both CM319 and UCH1 cell lines (Fig. 1c). These data indicated that the KRT8 expression of chordoma cells was significantly increased after chemotherapy.

\section{Doxorubicin or irinotecan induced UPR and autophagy in chordoma cells in vitro}

Human chordoma cells are characterized as large and vacuolized cells, and the notochordal vacuole is thought to be a lysosome-related organelle ${ }^{9,11,12,38-40}$. As lysosome organelles are often related to autophagy activity and ER stress $^{41,42}$, we further explored the UPR and autophagy flux after chemotherapy in chordoma cells. A significant increase of splicing of XBP1 mRNA was observed after treatment with Doxo $(0.5 \mu \mathrm{M})$ and Irino $(50 \mu \mathrm{M})$ for $12 \mathrm{~h}$ or $24 \mathrm{~h}$, as shown by RT-PCR, which indicated that the IRE1- $\alpha$ arm of the UPR was activated (Fig. 2a). In addition, the western blotting analysis (Fig. 2b) demonstrated a two-four folds increase of the expression of four main UPR-related proteins, BiP, CHOP (C/EBP Homologous Protein), and ATF4, ATF6 in both CM319 and UCH1 cell lines after treatment with Doxo $(0.5 \mu \mathrm{M})$ and Irino $(50 \mu \mathrm{M})$ for $24 \mathrm{~h}$. Furthermore, immunofluorescence analysis also confirmed an elevated ER stress in CM319 and $\mathrm{UCH} 1$ cells after treatment with Doxo $(0.5 \mu \mathrm{M})$ and Irino $(50 \mu \mathrm{M})$ for $24 \mathrm{~h}$, as indicated by an enhanced expression of CHOP and BiP (Fig. 2c). Besides, we also observed an enhanced autophagy activity from both CM319 and UCH1 cell lines after being treated with Doxo $(0.5 \mu \mathrm{M})$ and Irino $(50 \mu \mathrm{M})$ for $12 \mathrm{~h}$ or $24 \mathrm{~h}$, indicated by 


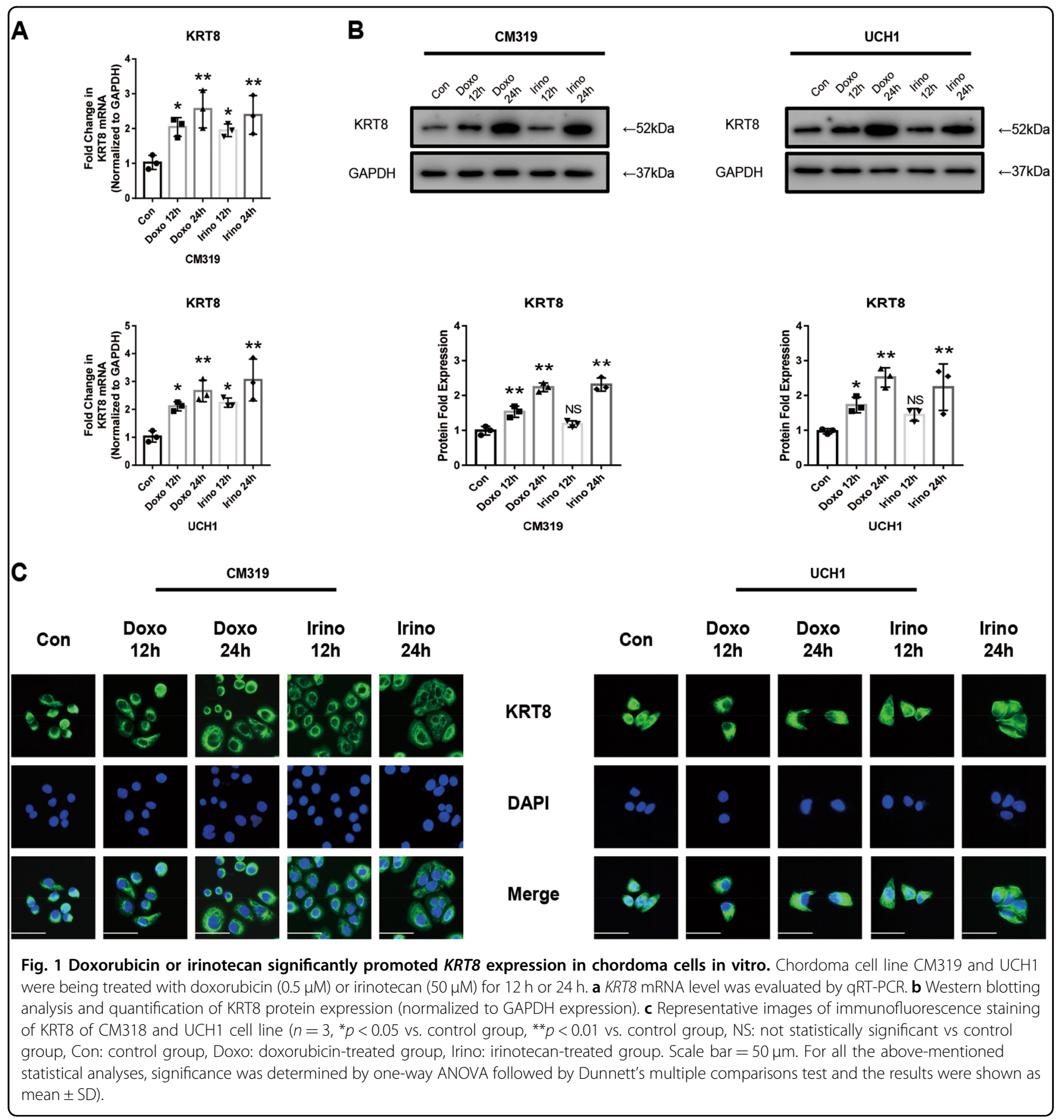

an increased expression of several autophagy-related genes $\operatorname{Atg} 7, B E C N 1$, and $L C 3 B$, at the mRNA level (Fig. 3a) and an increased conversion of LC3B I to LC3B II accompanied by a decreased expression of SQSTM1, as determined by the western blotting assay (Fig. 3b). Lastly, the immunofluorescence of LC3B also showed an increased LC3B puncta per cell in both CM319 and UCH1 cell lines after being treated with Doxo $(0.5 \mu \mathrm{M})$ and Irino $(50 \mu \mathrm{M})$ for $24 \mathrm{~h}$ (Fig. 3c). Collectively, these data showed that chemotherapy induced UPR and autophagy in chordoma cells.

Knockdown of KRT8 in chordoma cell increased its' sensitivity to chemotherapy by promoting its apoptosis in vitro

As the expression of KRT8 of chordoma cells was upregulated after chemotherapy, we hypothesized that $K R T 8$ plays a potential role in the chemoresistance of 


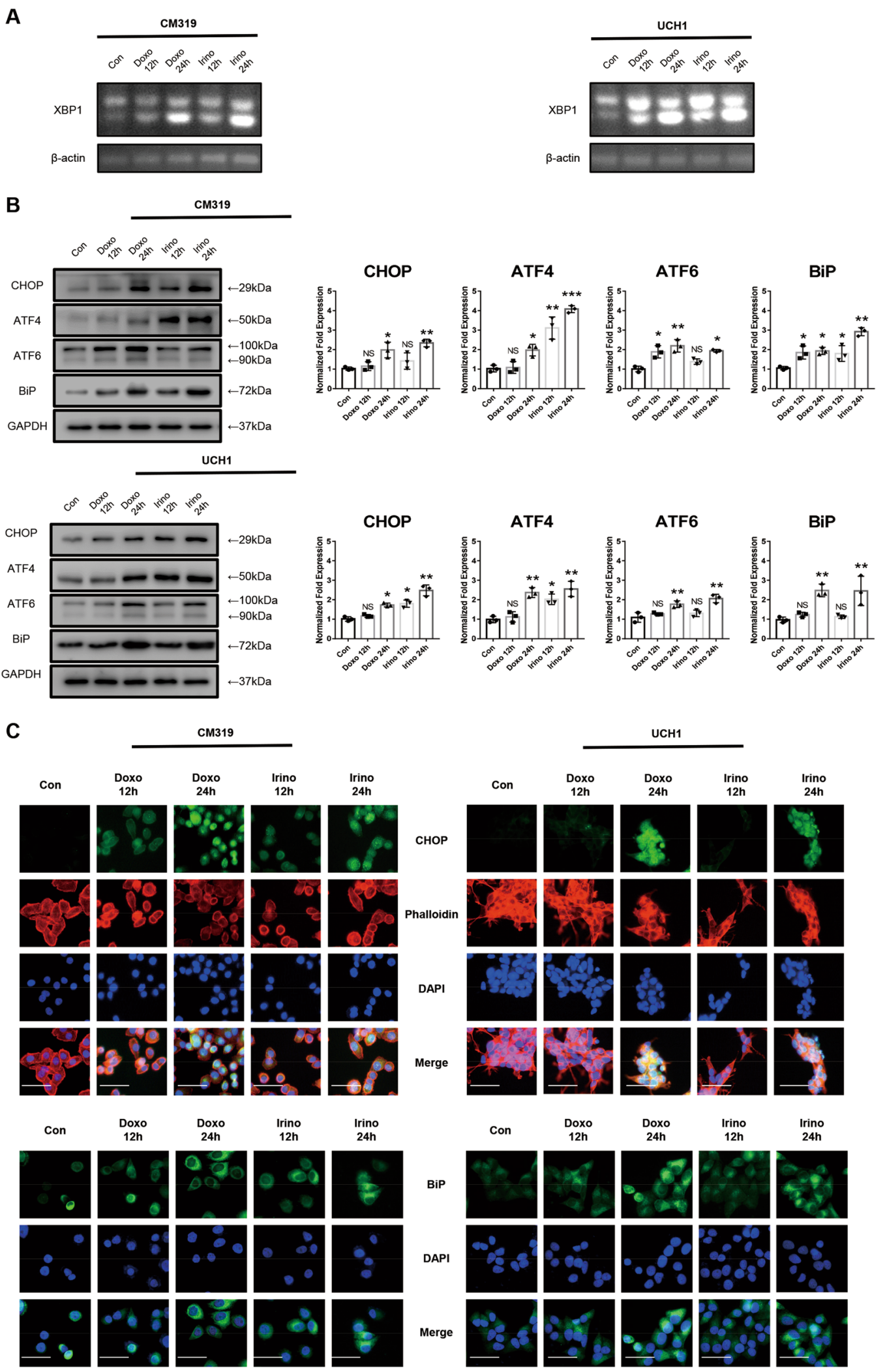

Fig. 2 (See legend on next page.) 
(see figure on previous page)

Fig. 2 Doxorubicin or irinotecan induced unfolded protein response in chordoma cells in vitro. Chordoma cell lines CM319 and UCH1 were being treated with doxorubicin $(0.5 \mu \mathrm{M})$ or irinotecan $(50 \mu \mathrm{M})$ for $12 \mathrm{~h}$ or $24 \mathrm{~h}$ in vitro. a Splicing of XBP1 mRNA were evaluated by RT-PCR. b Western blotting analysis and quantification of CHOP, ATF4, ATF6, and BiP protein expression (normalized to GAPDH expression). c Representative images of immunofluorescence staining of $\mathrm{CHOP}$ and BiP of $\mathrm{CM} 319$ and $\mathrm{UCH} 1$ cell line $\left(n=3,{ }^{*} p<0.05\right.$ vs. control group, ${ }^{* *} p<0.01$ vs. control group, NS: not statistically significant vs. control group, Con: control group, Doxo: doxorubicin-treated group, Irino: irinotecan-treated group. Scale bar $=50 \mu \mathrm{m}$. For all the above-mentioned statistical analyses, significance was determined by one-way ANOVA followed by Dunnett's multiple comparisons test and the results were shown as mean $\pm \mathrm{SD}$ ).

chordoma cells. We then used siRNA to knock down the KRT8 expression of chordoma cells, followed by chemotherapy in vitro. After transfection with siRNA for $24 \mathrm{~h}$, the qRT-PCR and western blotting analysis showed a significant decrease of KRT8 both at the mRNA and protein level (Fig. S1a, b). Then, we treated the chordoma cells with Doxo $(0.5 \mu \mathrm{M})$ or Irino $(50 \mu \mathrm{M})$ for $24 \mathrm{~h}$ after transfection with siRNA for $24 \mathrm{~h}$. First, the Cell Counting Kit 8 (CCK8) assay illustrated a significant decrease in cell viability in the "si + doxo" or "si + Irino" group, compared with the "Doxo" or "Irino" group, respectively. However, siKRT8 alone did not affect cell viability on both CM319 and UCH1 cell lines significantly (Fig. 4a). Then, as determined by western blotting analysis and immunofluorescence analysis, the expression of KRT8 was significantly decreased in "si + doxo" or "si + Irino" group, compared with "Doxo" or "Irino" group, respectively (Fig. 4b, c). In addition, we observed a significant increase in cleaved PARP and Caspase 4 protein expression level, which indicated an increase in ER stress-induced apoptosis (Fig. 4b). Then the Annexin V-PE (Phycoerythrin)/ propidium iodide (PI) staining measured by flow cytometry also confirmed a significant increased the apoptosis in "si + doxo" or "si + Irino" group, compared with "Doxo" or "Irino" group, respectively (Fig. 4d). Finally, another set of siRNA (siKRT8-2) was used to rule out the possible "off-target" effect of siRNA (Supplementary Fig. S2). These data illustrated that knockdown of KRT8 increased its sensitivity to chemotherapy of chordoma cells by promoting its apoptosis.

\section{Knockdown of KRT8 followed by chemotherapy promoted apoptosis of chordoma cells through aggregating ER stress through PERK/elF2 $a$ arm of UPR in vitro}

Disturbance of cellular homeostasis, which leads to accumulation of unfolded/misfolded protein inside the $\mathrm{ER}$, induces a cellular condition known as the ER stress and then triggers a set of intracellular signaling pathways collectively referred to as the UPR, to transcriptionally and translationally cope with the aberrant proteins ${ }^{25,43,44}$. As we have demonstrated that chemotherapy induced UPR in chordoma cells, we further investigated the activation of UPR in cases where KRT8 was knocked down, followed by chemotherapy. After transfection with siRNA for $24 \mathrm{~h}$, we treated the chordoma cells with Doxo
$(0.5 \mu \mathrm{M})$ or Irino $(50 \mu \mathrm{M})$ for $24 \mathrm{~h}$. The RT-PCR showed that there were no significant changes in splicing of XBP1 mRNA level in the "si + doxo" or "si + Irino" group, compared with the "Doxo" or "Irino" group, respectively (Fig. 5a). Consistent with RT-PCR results, there were no significant changes in XBP1-s protein expression level, as determined by western blotting analysis in these groups (Fig. 5b). Similar to that, the ATF6 protein expression level also showed no significant changes in these groups (Fig. 5b). However, as determined by western blotting analysis, the phosphorylation of PERK and eIF2 $\alpha$, the two major components of PERK/eIF2 $\alpha$ arm of the UPR, were strikingly increased in "si + doxo" or "si + Irino" group, compared with "Doxo" or "Irino" group, respectively (Fig. 5b). However, no significant changes in protein expression level of BiP, CHOP in "siKRT8" group, compared with "siNC" group, were observed (Supplementary Fig. S1b). These data clearly showed that knockdown of KRT8 followed by chemotherapy significantly aggregate ER stress through the PERK/eIF2 $\alpha$ arm of UPR.

\section{Knockdown of KRT8 followed by chemotherapy blocked the late stage of autophagy in vitro}

Recently, ample studies showed that enhanced autophagic responses can support cancer cell survival, proliferation, and growth in adverse microenvironmental conditions, such as the presence of chemotherapy, and therefore contributes to drug resistance ${ }^{32,36}$. As we have showed that chemotherapy could promote autophagy activity in chordoma cells, we further investigated the autophagy flux in cases where KRT8 was knocked down followed by chemotherapy. After transfected with siRNA for $24 \mathrm{~h}$, we treated the chordoma cells with Doxo $(0.5 \mu \mathrm{M})$ or Irino $(50 \mu \mathrm{M})$ for $24 \mathrm{~h}$. As determined by qRT-PCR, there were no significant changes in $\operatorname{Atg} 7$, $B E C N 1$, and $L C 3 B$ mRNA level in "si + doxo" or "si + Irino" group, compared with "Doxo" or "Irino" group, respectively (Fig. 6a). However, the western blotting analysis showed a significant higher LC3B II/I ratio in these groups (Fig. 6b). Consistent with western blotting results, the immunofluorescence of LC3B also showed a significant increase of LC3B puncta per cell in these groups (Fig. 6c). Moreover, as determined by western blotting, the SQSTM1 expression level was significantly increased in "si + doxo" or "si + Irino" group, compared 


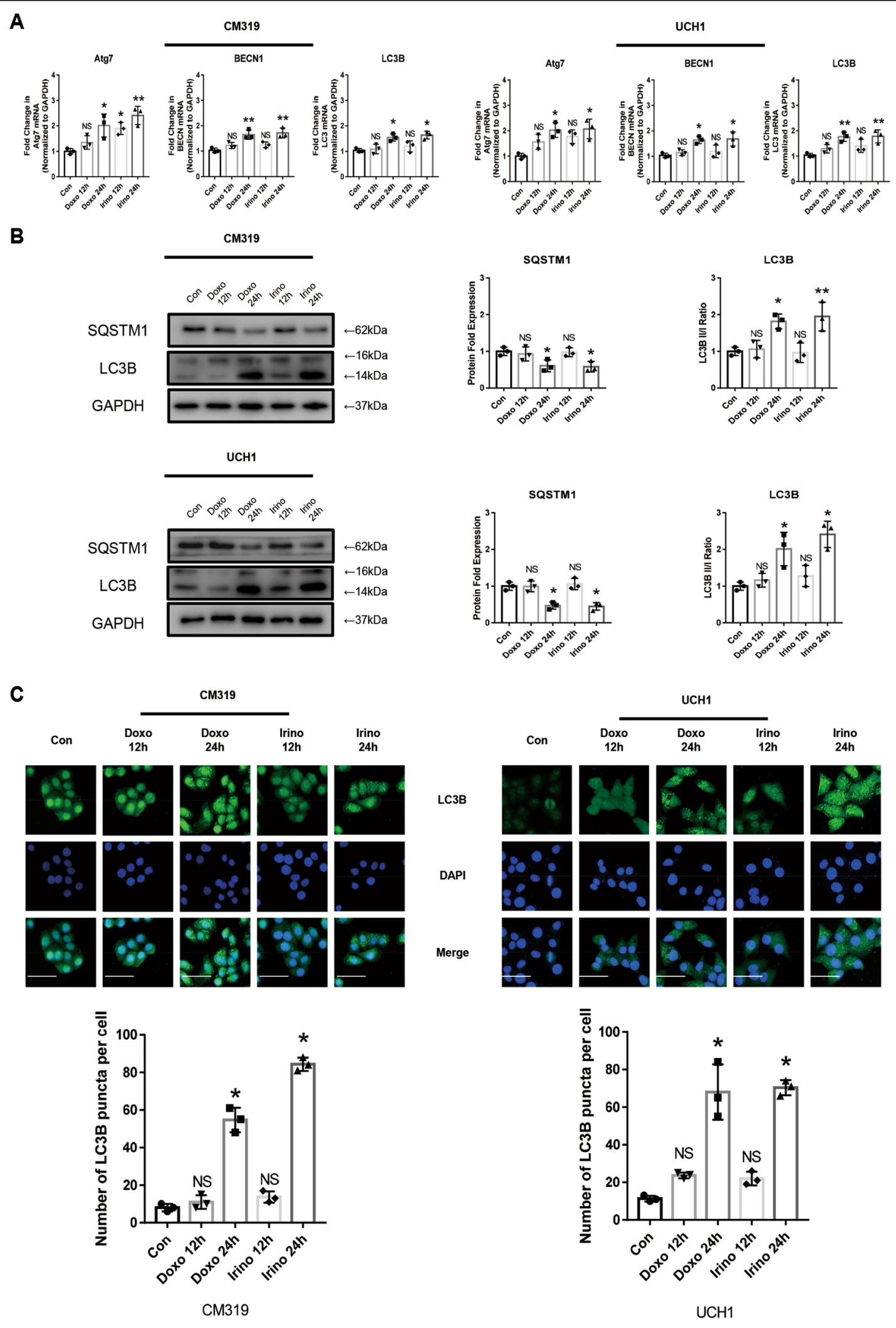

Fig. 3 (See legend on next page.) 
(see figure on previous page)

Fig. 3 Doxorubicin or irinotecan induced autophagy in chordoma cells in vitro. Chordoma cell line CM319 and UCH1 were being treated with doxorubicin $(0.5 \mu \mathrm{M})$ or irinotecan $(50 \mu \mathrm{M})$ for $12 \mathrm{~h}$ or $24 \mathrm{~h}$ in vitro. a Atg7, BECN1, and LC3B mRNA levels were determined by qRT-PCR. b Western blotting analysis and quantification of SQSTM1 and LC3B protein expression (normalized to GAPDH expression). c Immunofluorescence staining of LC3B and quantification of LC3B puncta per cell of CM319 and UCH1 cell line $\left(n=3,{ }^{*} p<0.05\right.$ vs. control group, ${ }^{* *} p<0.01$ vs. control group, NS: not statistically significant vs. control group, Con: control group, Doxo: doxorubicin-treated group, Irino: irinotecan-treated group. Scale bar $=50 \mu \mathrm{m}$. For all the above-mentioned statistical analyses, significance was determined by one-way ANOVA followed by Dunnett's multiple comparisons test and the results were shown as mean $\pm \mathrm{SD}$ ).

with "Doxo" or "Irino" group, respectively (Fig. 6b). However, no significant changes in protein expression level of LC3B, SQSTM1 in "siKRT8" group, compared with "siNC" group were observed (Fig. S1b). As LC3B I to LC3B II conversion occurred at an early stage of autophagy and SQSTM1 degradation occurred at a late stage of autophagy, the knockdown of KRT8 followed by chemotherapy induced a blockage of late-stage autophagy.

PERK inhibitor GSK2606414 decreased UPR activation and partially abolished chemosensitizing effect of siKRT8 but did not reverse the blockage of autophagy flux in vitro

To further elucidate the relationship between the elevated UPR and the blockage of autophagy flux in cases where knockdown of KRT8 was followed by chemotherapy, we used specific PERK inhibitor GSK2606414 (2 $\mu \mathrm{M})$ to inhibit the activation of PERK arm of UPR after knockdown of KRT8 and chemotherapy. Unsurprisingly, GSK2606414 abolished the activation of PERK/eIF2 $\alpha$ arm of UPR brought by knockdown of KRT8 followed by chemotherapy, as shown by decreased p-PERK and p-eIF2 $\alpha$ expression (Fig. 7a). Also, the inhibition of UPR significantly rescued the apoptosis of chordoma cells, as shown by decreased expression of cleaved PARP (Fig. 7a). Consistent with western blotting results, the Annexin VPE/PI staining measured by flow cytometry also confirmed a significant decreased apoptosis after treatment of GSK2606414 (Fig. 7b). However, we did not observe a significant change of LC3B II/I ratio and SQSTM1 protein expression after the inhibition of PERK/eIF $2 \alpha$ arm of UPR by GSK2606414 (Fig. 7a), which indicated that the blockage of autophagy was independent of the activation of UPR. These data showed that PERK inhibitor GSK2606414 decreased UPR activation and partially abolished chemosensitizing effect but did not reverse the blockage of autophagy flux brought up by the knockdown of KRT8.

\section{Knockdown of KRT8 increased chemosensitivity of chordoma cells in vivo}

As we have clearly showed that knockdown of KRT8 overcomes chemoresistance of chordoma cells by promoting its apoptosis in vitro, to further examine the chemosensitizing effect of siKRT8 in vivo we developed a xenograft model using the CM319 cell line. Briefly,
CM319 cells were subcutaneously injected into NOD/ SCID (Non-obese diabetic/scid gamma) mice. When the mean tumor volume reached $500 \mathrm{~mm}^{3}$ (15 days after the injection of CM319 cells), the NOD/SCID mice were randomly assigned to four groups: three mice for each group-control group (Con), siKRT8-treated group (siKRT8), Doxo (2 mg/kg), and siKRT8 and Doxo group (si + Doxo $2 \mathrm{mg} / \mathrm{kg}$ ). Doxo were intraperitoneally administered every day for 14 days and siRNA was transfected three times weekly. After 30 days from the injection of the CM319 cells, the mice were killed and xenografts were collected for weighing and histology analysis. The results showed that the "si + Doxo" treatment resulted in a significant inhibition on tumor growth compared with the "Doxo" group and control group (Fig. 8a); yet, the "siKRT8" group showed no significant changes in terms of tumor growth (Fig. 8a). Western blotting analysis showed a significant increase in $\mathrm{BiP}, \mathrm{CHOP}$, Caspase 4 protein expression in "si + Doxo" group, compared with control group or "si + Doxo" group, which indicated a promotion of ER stress-induced cell death (Fig. 8b). Also, the western blotting analysis showed an increased SQSTM1 protein expression level in the "si + Doxo" group compared with the "Doxo" group, which indicated a blockage of autophagy flux (Fig. 8b). However, the "siKRT8" group showed no significant changes in protein expression of $\mathrm{BiP}$, CHOP, Caspase 4, SQSTM1, and LC3B, compared with the control group (Fig. 8b). Immunofluorescence staining of tumor sections also confirmed an increase in BiP expression in the "si + Doxo" group compared with the "Doxo" group and control group (Fig. 8c). Consistently, transmission electron microscopic analysis of tumor tissue showed aberrant distension of the ER in the "si+ Doxo" group compared with the control group and "Doxo" group (Fig. 8d). These data showed that knockdown of KRT8 increased chemosensitivity of chordoma cells through aggravating ER stress and blocking latestage autophagy in vivo.

\section{Discussion}

Chordoma is originated from notochord remnants, which were characterized as large vacuolized cells ${ }^{13,45,46}$. Its high insensitivity to chemotherapy and the lack of curative therapy warrant the investigation into new therapeutic approaches ${ }^{4,6}$. Doxo is a classical topoisomerase II 


$$
\text { A }
$$

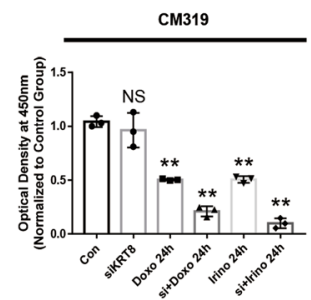

B

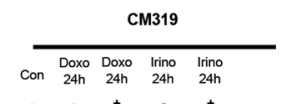

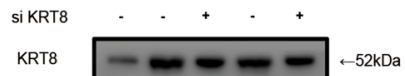

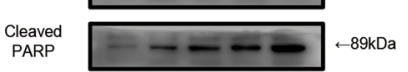

Caspase $4-----43 \mathrm{kDa}$

GAPDH $----\infty 7 \mathrm{kDa}$

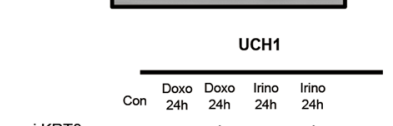
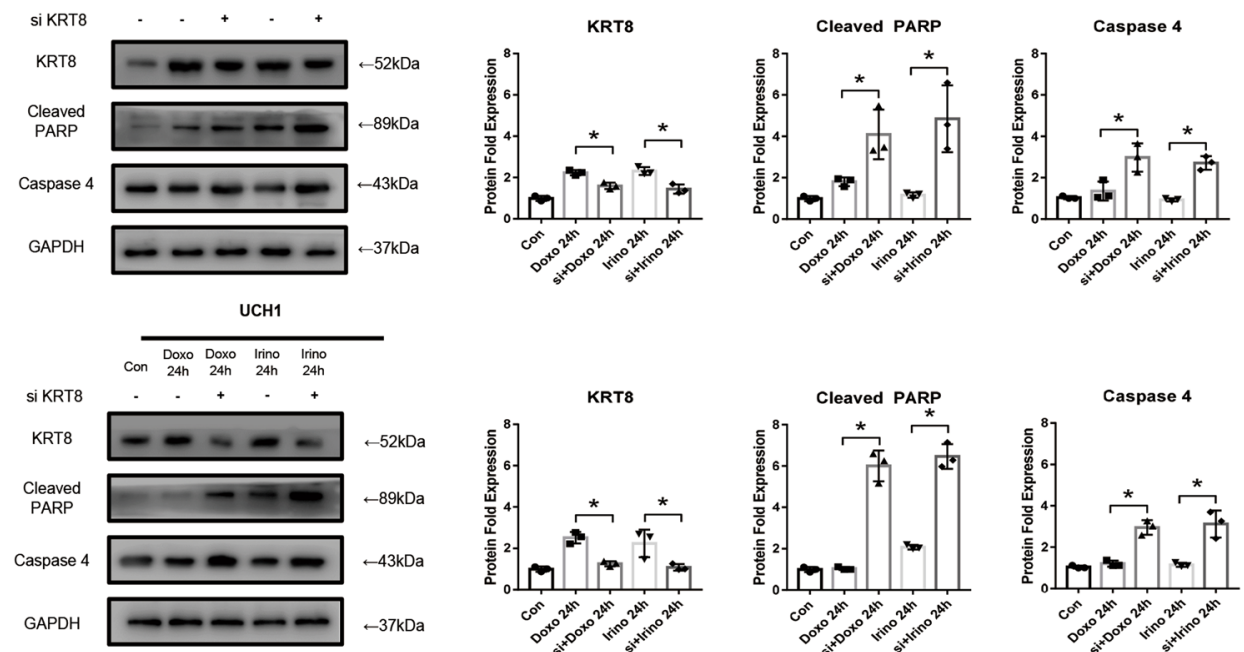

C
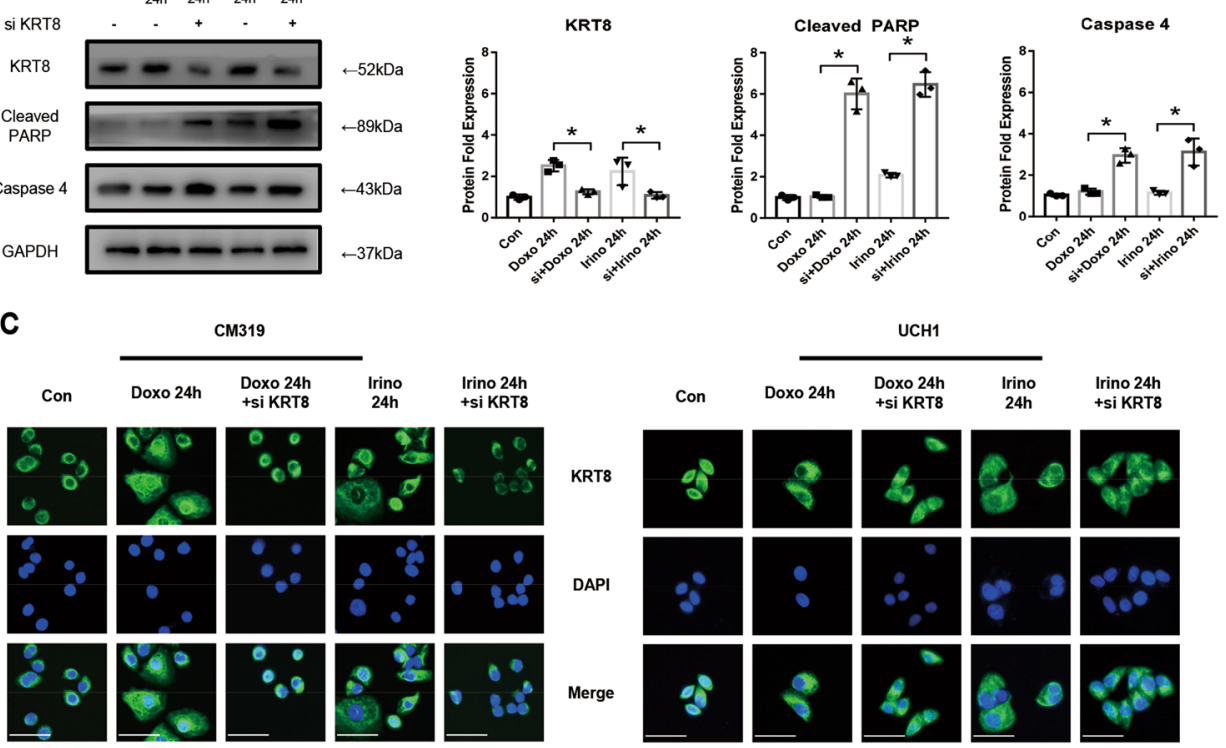

Di KRT8

con

\section{.}

$\begin{array}{ll}0 & 0 \\ 0 & 0 \\ & \end{array}$

$\frac{0_{0}^{\circ}}{0_{0}^{\circ}}$

0
0

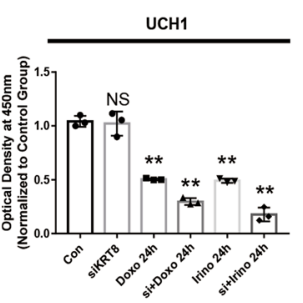

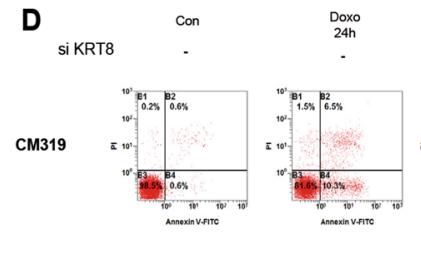
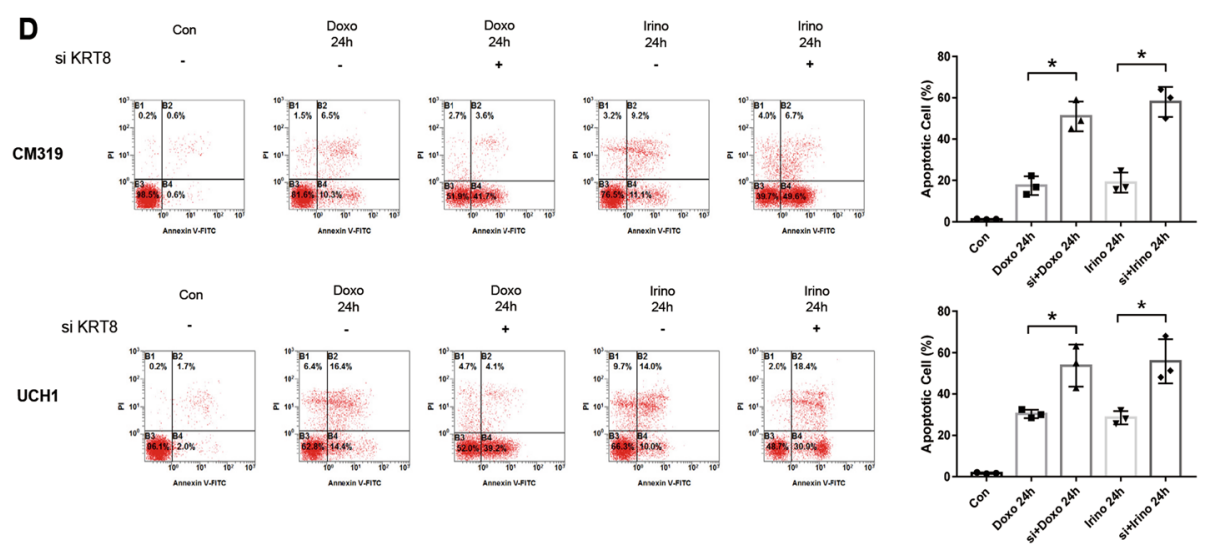

Fig. 4 (See legend on next page.) 
(see figure on previous page)

Fig. 4 Knockdown of KRT8 in chordoma cell increased its' sensitivity to chemotherapy by promoting its apoptosis in vitro. Chordoma cell line CM319 and UCH1 were transfected with siKRT8 followed by treatment of doxorubicin ( $0.5 \mu \mathrm{M})$ or irinotecan $(50 \mu \mathrm{M})$ for $24 \mathrm{~h}$. a Cell viability of chordoma cells were determined by CCK8 assay. b Western blotting analysis and quantification of KRT8, cleaved PARP, and Caspase 4 protein expression (normalized to GAPDH expression, quantification data of KRT8 in the "Doxo $24 \mathrm{~h}$ " group and "Irino $24 \mathrm{~h}$ " group were derived from the same data set in Fig. 1b). c Immunofluorescence staining of KRT8 of CM319 and UCH1 cell line. $\mathbf{d}$ Apoptosis of chordoma cells was determined by Annexin V-PE/PI staining measured by flow cytometry $\left(n=3,{ }^{*} p<0.05\right.$ vs. indicated group, ${ }^{* *} p<0.01$ vs. indicated group, NS: not statistically significant vs. indicated group, Con: control group, Doxo: doxorubicin-treated group, Irino: irinotecan-treated group. Scale bar $=50 \mu \mathrm{m}$. For all the above-mentioned statistical analyses, significance was determined by one-way ANOVA followed by Tukey's multiple comparisons test and the results were shown as mean \pm S.D.).

inhibitor, which chordoma cells are relatively sensitive to $^{38,39}$. Irino, a topoisomerase I inhibitor, is one of the camptothecin analogs that has been approved for cancer treatment and is the only chemotherapy agent that has finished phase II study on chordoma ${ }^{4,47}$. In this study, we found that either $0.5 \mu \mathrm{M}$ Doxo or $50 \mu \mathrm{M}$ Irino induce apoptosis of chordoma cells, after being treated for $24 \mathrm{~h}$. However, a difference of chemoresistance between CM319 and UCH1 cell lines is observed. As determined by Annexin V-PE/PI apoptosis assay, CM319 cell line is more resistant to Doxo than UCH1 cell line; yet, both CM319 and UCH1 cell lines show a similar sensitivity to Irino.

Keratins have a cell-specific expression pattern, which is employed to distinguish different epithelial cell types. KRT8 expresses in many tissues, including the liver, retina, pancreas, and intestine, executing multiple cellular functions, including mechanical support, stem cell-fate control, and cytoprotective response ${ }^{10,14,15,17-20,22}$. Pekny et al. $^{48}$ reported that keratins were upregulated during or recovering from stress. The study conducted by $\mathrm{Ku}$ and Omary ${ }^{15}$ showed that KRT8 could serve as a "phosphate sponge" and thus protect liver cells from stress-induced apoptosis. In addition, Lähdeniemi et al. ${ }^{19}$ demonstrated that KRT8 and Notch1 colocalized and interacted with each other in the colonic epithelial cells. KRT8 could also enhance Notch1 protein levels and activity in a dosedependent manner and regulate Notch1 signaling activity and differentiation in the epithelium of the large intestine ${ }^{19}$. Also, a previous study conducted by Trisdale et al. $^{22}$ showed an increased FAS expression and a decreased resistance to Fas-activating antibody after knockdown of KRT8 in granulosa cell tumors. Consistent with their results, we also find $K R T 8$ plays an important role in chordomas' apoptosis resistance. However, we do not observe an increased expression of FAS after knockdown of KRT8 alone or followed by treatment of Doxo or Irino as determined by qRT-PCR, and this may due to the low expression of FAS in chordoma cells (data not shown). The data from this study show that KRT8 is significantly upregulated after treatment with Doxo and Irino for $24 \mathrm{~h}$. Moreover, immunocytochemistry analysis further demonstrates that KRT8 expression is promoted throughout the cell in both CM319 and UCH1 cell lines.
Then, our data show that siRNA-mediated knockdown of $K R T 8$ alone do not promote cell apoptosis, nor does it induce UPR and autophagy in chordoma cells. However, after being treated with Doxo or Irino, strikingly increased apoptosis is observed. Collectively, in this study, we show that KRT8 executes a cytoprotective action during chemotherapy and endows chordoma cells with greater chemoresistance. In addition, knockdown of KRT8 sensitizes both CM319 and UCH1 cell lines to both Doxo and Irino, which shows that KRT8 may serve as a universal therapeutic target for chemoresistant chordoma.

The ER fulfills multiple cellular functions. A number of stresses, including nutrient deprivation, high metabolic demand, and chemotherapies, disturbed the proteinfolding capacities within the ER, thus triggering an evolutionarily conserved response, collectively referred to as UPR. The UPR is considered as a significant factor in many pathologies, including cancer, liver dysfunction, neurodegenerative diseases, and ischemia ${ }^{27,49-52}$. To date, emerging evidence from many studies have revealed that activation of UPR endows tumor cells with great drugresistant capacity. In general, the activation of UPR initially favors a cytoprotective response; however, it also paradoxically induces apoptosis when cells fail to adapt to severe ER stress ${ }^{25,53-55}$. In this study, we find that Doxo or Irino induce a moderate UPR in chordoma cells as shown by an increased expression of CHOP, BiP, ATF4, ATF6, and XBP1-s. In addition, a difference of the arms of UPR induced was observed between Doxo and Irino. As shown by western blotting analysis, Doxo preferentially induces ATF6 arm of UPR, whereas Irino preferentially induces ATF4 arm of UPR, in both CM319 and UCH1 cell lines. This discrepancy may be explained by the different pharmacological action of these two drugs (Irino is a topoisomerase I inhibitor, whereas Doxo is a topoisomerase II inhibitor). Then we further investigate the role of KRT8 during UPR and the data show that siRNAmediated knockdown of KRT8 alone does not provoke significant changes in UPR. However, after treatment with Doxo or Irino, a strikingly elevated expressions of CHOP, $\mathrm{BiP}$ are observed, which represents an increase in ER stress. Moreover, we also find that knockdown of KRT8 


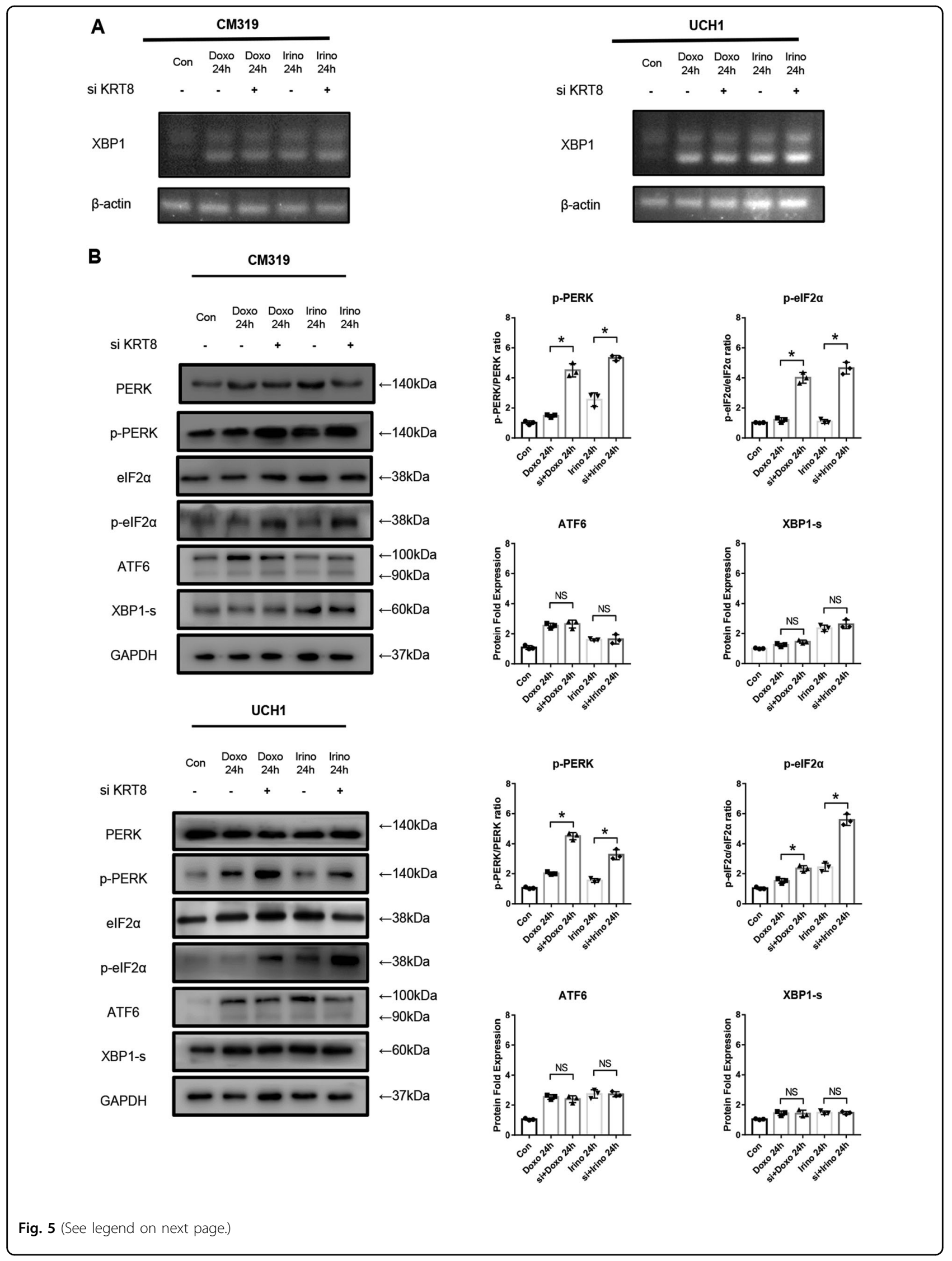


(see figure on previous page)

Fig. 5 Knockdown of KRT8 followed by chemotherapy promoted apoptosis of chordoma cells through aggregating ER stress through PERK/elF2 $\boldsymbol{a}$ arm of UPR in vitro. Chordoma cell line CM319 and UCH1 were transfected with siKRT8 followed by treatment of doxorubicin ( $0.5 \mu M)$ or irinotecan $(50 \mu \mathrm{M})$ for $24 \mathrm{~h}$. a Splicing of XBP1 mRNA were evaluated by qRT-PCR. $\mathbf{b}$ Western blotting analysis and quantification of PERK, p-PERK, elF2a, p-elF2a, ATF6, and XBP1-s protein expression (p-PERK and p-elF2a were normalized to PERK and elF2a expression, respectively; ATF6 and XBP1$s$ were normalized to GAPDH expression; quantification data of ATF6 in "Doxo $24 \mathrm{~h}$ " group and "Irino $24 \mathrm{~h}$ " group was derived from the same data set in Fig. $2 b)\left(n=3,{ }^{*} p<0.05\right.$ vs. indicated group, ${ }^{* *} p<0.01$ vs. indicated group, NS: not statistically significant vs. indicated group, Con: control group, Doxo: doxorubicin-treated group, Irino: irinotecan-treated group. For all the above-mentioned statistical analyses, significance was determined by one-way ANOVA followed by Tukey's multiple comparisons test and the results were shown as mean \pm SD).

aggravates ER stress through the PERK/eIF2 $\alpha$ arm of UPR, as shown by an increased expression of p-PERK and p-eIF2 $\alpha$ expression.

Autophagy can be induced by nutrient deprivation, ER stress, and chemotherapy, functioning as a pro-survival strategy. Ample studies and clinical trials supported autophagy as a potential yet potent therapeutic target for many drug-resistant malignant tumors ${ }^{34,35,37,56}$. The large vacuole of chordoma cells are considered as a lysosomerelated organelle and some studies tried to link these large vacuoles to drug-resistant capacity by targeting autophagosome-lysosome fusion using chloroquine, an effective anti-malaria drug. However, the results showed that chloroquine only exerted a weak growth inhibition on chordoma cells and could only serve as a weak chemosensitizer for doxorubicin. Moreover, this chemosensitizing effect was only observed in the MUG-Chor1 cell line but not in the MUG-CC1 cell line; this may be because the autophagy flux of chordoma cells cannot be inhibited within the therapeutic concentration window of chloroquine. Despite this, Kolb-Lenz et al. ${ }^{38}$ still believed that the huge lysosomal mass of chordoma might in fact represent a drug target, which is worth testing in further studies. The connection between KRT8 and autophagy has been reported. Kongara et al. $^{57}$ showed that high phosphor (Ser73)-KRT8 levels are inversely correlated with Beclin 1 expression in human breast tumor cells. Furthermore, phosphor (Ser73)-KRT8 was accumulated in metabolically stressed immortalized mammary epithelial cells in a SQSTM1-dependent manner upon autophagy inhibition ${ }^{57}$. In addition, Baek et al. ${ }^{16}$ showed that knockdown of KRT8 affected the fusion between autophagosomes and lysosomes after being treated with paraquat, and increased expression of KRT8 following autophagy provides a cytoprotective role in retinal pigment epithelium. Consistently, our data show an elevated autophagy activity within the chordoma cells after chemotherapy. Moreover, knockdown of KRT8, followed by treatment with Doxo or Irino, prevents SQSTM1 from degradation as shown by western blotting, which indicates that the late stage of autophagy is blocked. In addition, we further elucidate that the blockage of autophagy flux brought by KRT8 knockdown is independent of the activation of the PERK/eIF2 $\alpha$ arm of UPR, as no significant change in SQSTM1 expression is observed, after treatment of PERK inhibitor GSK2606414. However, despite the fact that the PERK inhibitor GSK2606414 abolishes the elevated UPR and partially rescues chordomas' apoptosis, the apoptotic ratio is still significantly higher than the "Doxo" or "Irino" group, as determined by two-tailed unpaired Student's $t$-test (data not shown). A most likely explanation is that knockdown of KRT8 successfully blocks the late-stage autophagy and therefore chemosensitizes chordoma cells.

To conclude, in the present study, we find that KRT8 is upregulated after treatment with Doxo and Irino. In addition, upregulated $K R T 8$ is accompanied by induction of autophagy and UPR. Knockdown KRT8 chemosensitizes chordoma by strikingly aggravating ER stress through the PERK/eIF2 $\alpha$ arm of UPR and blocks the latestage autophagy. In addition, the blockage of late-stage autophagy is independent of the activation of the PERK/ eIF2 $\alpha$ arm of UPR. Lastly, a xenograft model using CM319 cell line further confirms that knockdown of KRT8 increases chemosensitivity of chordoma in vivo. This study represents the first systematic investigation into the role of KRT8 in chemoresistance of chordoma cells and our results suggest $K R T 8$ as a promising therapeutic target for chemoresistant chordoma.

\section{Materials and methods \\ Cell culture and reagents}

Human chordoma cell line CM319 $9^{2,58}$ was a gift from Dr. Wen Yan-hua (Department of Orthopedic Oncology, Tang Du Hospital, Xi'an, China) and was maintained at $37^{\circ} \mathrm{C}$ under $5 \% \mathrm{CO}_{2}$ in RPMI 1640 medium (Gibco, USA) supplemented with $10 \%$ fetal bovine serum (FBS) (Gibco, USA), penicillin (100 units/ml), and streptomycin $(100 \mu \mathrm{g} / \mathrm{ml})$. Human chordoma cell line $\mathrm{UCH} 1^{13,39,40}$ was obtained from American Type Culture Collection (\#CRL3217) and was maintained in rat tail type I collagen (BD Biosciences)-coated flasks at $37^{\circ} \mathrm{C}$ under $5 \% \mathrm{CO}_{2}$ in Iscove/RPMI (4:1) medium (Gibco, USA), supplemented with 10\% FBS (Gibco, USA), penicillin (100 units/ml), streptomycin $(100 \mu \mathrm{g} /)$, and $1 \%(\mathrm{v} / \mathrm{v})$ L-glutamine (Gibco, USA). Doxo (\#HY-15142A), Irino (\#HY-16562), and 


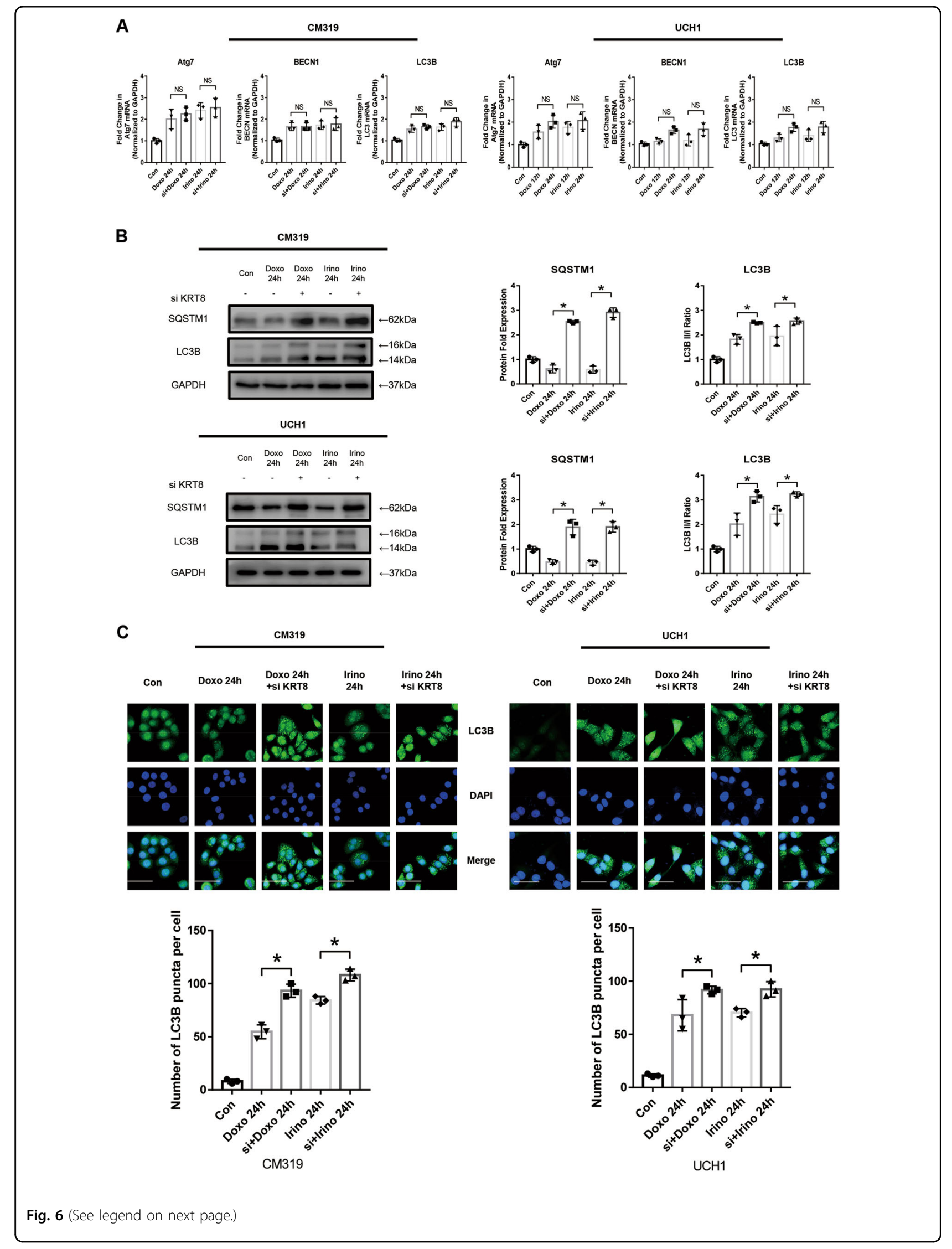


(see figure on previous page)

Fig. 6 Knockdown of KRT8 followed by chemotherapy blocked the late stage of autophagy in vitro. Chordoma cell line CM319 and UCH1 were transfected with siKRT 8 followed by treatment of doxorubicin $(0.5 \mu \mathrm{M})$ or irinotecan $(50 \mu \mathrm{M})$ for $24 \mathrm{~h}$. a Atg7, BECN1, and LC3B mRNA level were determined by qRT-PCR. $\mathbf{b}$ Western blotting analysis and quantification of SQSTM1 and LC3B protein expression (normalized to GAPDH expression, quantification data of LC3B and SQSTM1 in "Doxo $24 \mathrm{~h}$ " group and "Irino $24 \mathrm{~h}$ " group was derived from the same data set in Fig. 3b).

c Immunofluorescence staining of LC3B and quantification of LC3B puncta per cell of CM319 and UCH1 cell line (quantification data of LC3B puncta in "Doxo $24 \mathrm{~h}$ " group and "Irino $24 \mathrm{~h}$ " group was derived from the same data set in Fig. $3 c)\left(n=3,{ }^{*} p<0.05\right.$ vs. indicated group, ${ }^{* *} p<0.01$ vs. indicated group, NS: not statistically significant vs. indicated group, Con: control group, Doxo: doxorubicin-treated group, Irino: irinotecan-treated group. Scale bar $=50 \mu \mathrm{m}$. For all the above-mentioned statistical analyses, significance was determined by one-way ANOVA followed by Tukey's multiple comparisons test and the results were shown as mean \pm SD).

GSK2606414 (\#HY-18072) were purchased from MedChemExpress (Shanghai, China). The final concentration of dimethylsulfoxide is $<0.05 \%(\mathrm{v} / \mathrm{v})$. No mycoplasma was detected in the CM319 and UCH1 cell lines.

\section{siRNA transfection in vitro}

The siRNA duplexes targeting human KRT8 and negative control (NC) siRNA were designed and synthesized by Genepharma (Suzhou, China). siRNA sequences used in this study were shown in Supplementary Table 1. siRNA transfection was performed using Lipofectamine 2000 Reagent (Invitrogen, USA) according to the manufacturer's instructions. Briefly, chordoma cells were seeded $\left(2.5 \times 10^{5}\right)$ in a six-well plate, after adhesion, and the cells were incubated with Optic-MEM medium (Thermo Fisher Scientific, USA, \#31985062) at $37^{\circ} \mathrm{C}$ for $2 \mathrm{~h}$. Cells were then transfected with $100 \mathrm{nM}$ of KRT8 siRNA or NC siRNA using Lipofectamine 2000 Reagent (Invitrogen, USA) and were incubated at $37^{\circ} \mathrm{C}$ for $4-6 \mathrm{~h}$. Finally, cells were washed with phosphate-buffered saline (PBS) and changed the medium with complete culture medium. Cells were collected for qRT-PCR or western blotting, to evaluate the knockdown efficacies $24 \mathrm{~h}$ later. In some experiments, knockdown of KRT8 was followed by treatment with Doxo, Irino, or GSK2606414.

\section{Cell viability assay}

Cell viability was evaluated by CCK8 (Dojindo, Kumamoto, Japan) according to the manufacturer's instructions. Briefly, CM319 and UCH1 cells were seeded into 96-well plates at a density of 5000/well with 3 replicates. After adhesion, the cells were treated accordingly. Then the original medium was replaced by a mixture of $10 \mu \mathrm{l}$ CCK8 reagent and $100 \mu \mathrm{l}$ fresh medium, and were incubated at $37^{\circ} \mathrm{C}$ for $4 \mathrm{~h}$. Finally, the optical density of each well was measured by a microplate reader (BioTek, USA) at $450 \mathrm{~nm}$.

\section{Flow cytometry apoptosis assay}

Chordoma cells were seeded in to a six-well plate and treated with indicated chemicals for desired period. After treatment, the chordoma cells were collected in $0.25 \%$ trypsin and washed three times with cold PBS. The apoptosis of chordoma cells was evaluated by Annexin VPE/PI apoptosis detection kit (BD Biosciences, USA) by flow cytometry.

\section{Western blotting analysis}

Chordoma cells or tumor tissue were collected and lysed in radioimmune precipitation assay buffer (Beyotime Biotechnology, China) containing a complete protease inhibitor cocktail (Roche, Germany), and the concentrations of protein were determined by Pierce BAC Protein Assay Kit (Thermo Fisher Scientific, USA). A certain amount of protein was mixed with SDSpolyacrylamide gel electrophoresis (PAGE) loading buffer (Beyotime Biotechnology, China), boiled for $15 \mathrm{~min}$, and subjected to SDS-PAGE followed by transferring to polyvinylidene difluoride (PVDF) membranes (Merck Millipore, Germany). Blots were probed with primary antibodies at $4{ }^{\circ} \mathrm{C}$ overnight, including the following: anti-KRT8 antibody (Abcam, \#ab53280, 1:1000), anti-LC3B (Abcam, \# ab48394, 1:500), antiSQSTM1 (Cell Signaling Technology, \#5114, 1:1000), anti-PERK (Cell Signaling Technology, \#3192, 1:1000), anti-p-PERK (Invitrogen, \#PA5-40294, 1:1000), antieIF2 $\alpha$ (Cell Signaling Technology, \#5324, 1:1000), antip-eIF2 $\alpha$ (Cell Signaling Technology, \#3398, 1:500), anti-Caspase 4 (Cell Signaling Technology, \#4450, 1:1000), anti-CHOP (Cell Signaling Technology, \#7351, 1:1000), anti-XBP1s (Cell Signaling Technology, \#40435, 1:500), anti-cleaved PARP (Santa Cruz Biotechnology, \#56196, 1:500), anti-BiP (Santa Cruz Biotechnology, \#13539, 1:1000), and anti-glyceraldehyde 3-phosphate dehydrogenase (GAPDH)f (Cell Signaling Technology, $\# 2118$, 1:1000). Then, incubate the membrane with horseradish peroxidase (HRP)-linked goat anti-rabbit IgG or horse anti-mouse IgG secondary antibody (Cell Signaling Technology, \#7074 or \#7076, Cell Signaling Technology) for $2 \mathrm{~h}$. Each step was followed by three washings in TBS (Tris-Buffered Saline) containing 0.1\% Tween-20 for $15 \mathrm{~min}$. Finally, the PVDF membranes were detected using Immobilon Western Chemiluminescent HRP Substrate (\#WBKLS0100, Millipore Corporation, Germany) and were observed under Amersham Imager 600 (General Electric, USA). 


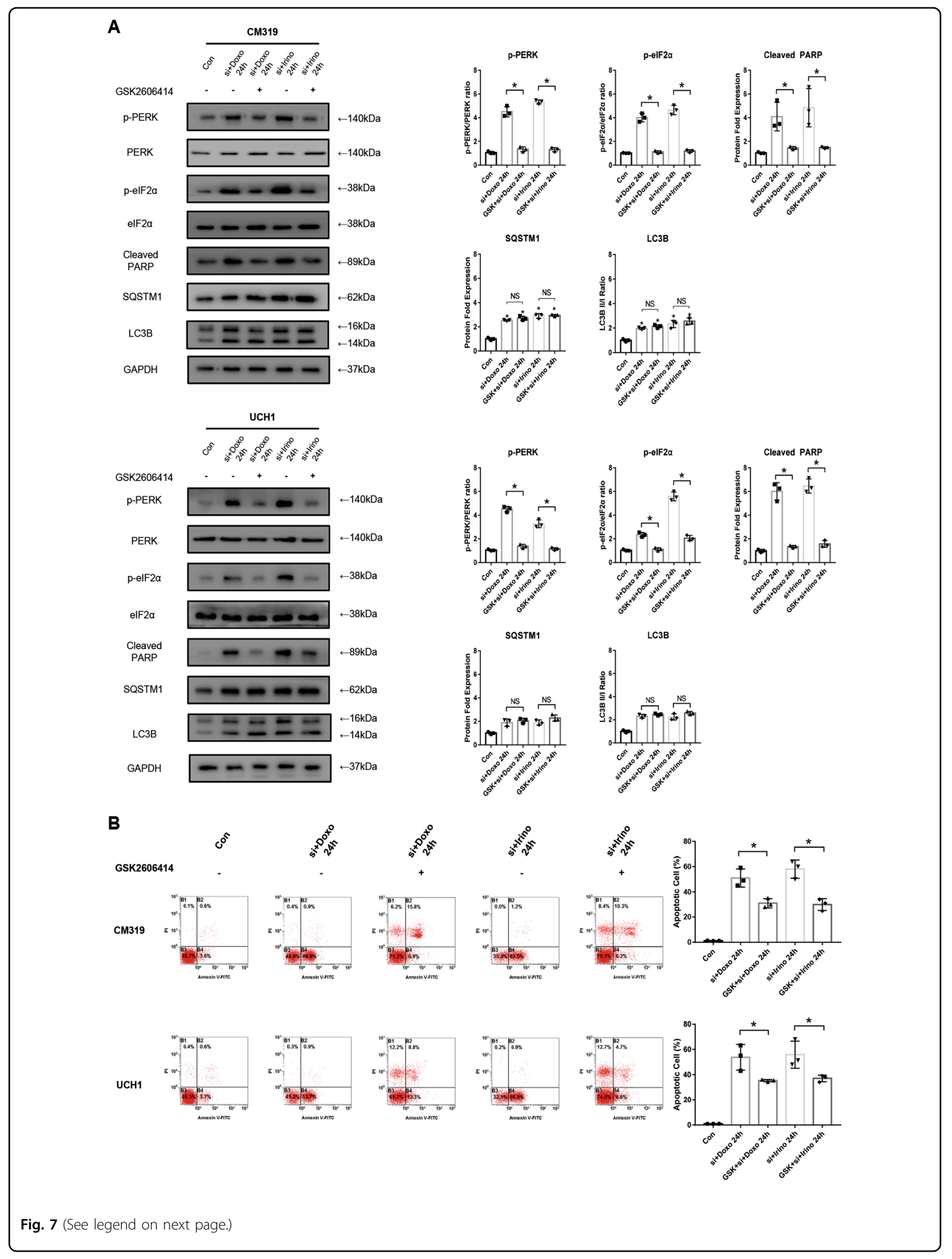


(see figure on previous page)

Fig. 7 PERK inhibitor GSK2606414 decreased UPR activation and partially abolished chemosensitizing effect of siKRT8 but did not reverse the blockage of autophagy flux in vitro. Chordoma cell line CM319 and UCH1 were transfected with siKRT8 followed by treatment of doxorubicin $(0.5 \mu \mathrm{M})$, irinotecan $(50 \mu \mathrm{M})$, and GSK2606414 $(2 \mu \mathrm{M})$ for $24 \mathrm{~h}$. a Western blotting analysis and quantification of p-PERK, p-elF2a, Cleaved PARP, SQSTM1, and LC3B protein expression (p-PERK and p-eIF2a were normalized to PERK and elF2a expression, respectively; Cleaved PARP, LC3B, and SQSTM1 were normalized to GAPDH expression; quantification data of p-PERK, p-elF2a, Cleaved PARP, SQSTM1, and LC3B in "Si + Doxo 24 " h" group and "si + Irino 24 h" group were derived from the same data set in Figs. $4 \mathrm{~b}$ and 5b). b Apoptosis of chordoma cells was determined by Annexin V-PE/ PI staining measured by flow cytometry (quantification data of apoptosis in "si + Doxo 24 h" group and "si + Irino 24 h" group were derived from the same data set in Fig. 4 d) $\left(n=3,{ }^{*} p<0.05\right.$ vs. the indicated group, ${ }^{* *} p<0.01$ vs. the indicated group, NS: not statistically significant vs. indicated group, Con: control group, Doxo: doxorubicin-treated group, Irino: irinotecan-treated group. For all the above-mentioned statistical analyses, significance was determined by one-way ANOVA followed by Tukey's multiple comparisons test and the results were shown as mean \pm SD).

\section{Quantitative qRT-PCR analysis}

Total RNA of chordoma cells were collected using MiniBEST Universal RNA Extraction Kit (TaKaRa, Japan) according to the manufacturer's instructions. Reverse transcription was performed with PrimeScript RT Master Mix (TaKaRa, Japan). Synthesized cDNA was then subjected to quantitative PCR analysis using TB Green Premix Ex Taq II (TaKaRa, Japan). The reactions were performed with CFX96 (Bio-Rad, USA). Gene expression levels were reported as relative fold change, with GAPDH as an internal control. Primers sequences used for qRT-PCR were shown in Supplementary Table 2.

\section{Immunofluorescence staining}

Chordoma cells were fixed in freshly prepared $4 \%$ paraformaldehyde for $30 \mathrm{~min}$ and permeabilized by $0.1 \%$ Triton X-100 (MP Biomedicals, USA) for $30 \mathrm{~min}$. Then the cells were and blocked by $0.8 \%$ bovine serum albumin in PBS for $1 \mathrm{~h}$, incubated with primary antibody anti:LC3B (Abcam, \#ab48394, 1:50), anti-CHOP (Cell Signaling Technology, \#7351, 1:50), anti-BiP (Santa Cruz Biotechnology, \#13539, 1:50), and anti-KRT8 (Abcam, \#ab53280, 1:50) at $4{ }^{\circ} \mathrm{C}$ overnight. Then cells were incubated with fluorescein isothiocyanate (FITC)-conjugated Affinipure Goat Anti-Mouse IgG(H $+\mathrm{L}$ ) (\#SA00003-1, Proteintech, China) or FITC-conjugated Affinipure Goat Anti-Rabbit $\operatorname{IgG}(\mathrm{H}+\mathrm{L})$ (\#SA00003-2, Proteintech, China) at room temperature for $2 \mathrm{~h}$. Finally, the cells were incubated with 4',6-diamidino-2-phenylindole (C1006, Beyotime Biotechnology, China) for 10 min or rhodamine phalloidin (\#PHDR1, Cytoskeleton, Inc, USA) for $30 \mathrm{~min}$. Each step was followed by washing with PBS three times for $5 \mathrm{~min}$ each. Then the cells or sections were analyzed under a fluorescence microscope (BX53, OLYMPUS, Japan). For tumor sections, antigen retrieval was performed with citrate buffer $(\mathrm{pH}=6.0)$.

\section{RT-PCR analysis}

To evaluate relative splicing level of XBP1 mRNA, RTPCR analysis was performed using SanTaq PCR Master Mix (Sangon, China). PCR products were analyzed on a
3.5\% agarose gel. Primers sequences used for qRT-PCR were shown in Supplementary Table 3.

\section{Mice xenograft model and siRNA transfection in vivo}

All animal experiments were approved and followed the guidelines issued by the Animal Experiment Administration Committee of the Fourth Military Medical University. Twelve 5-week-old male NOD/SCID mice were maintained under specific pathogen-free conditions. CM319 chordoma cells were collected and washed three times with cold PBS, then $100 \mu \mathrm{l}$ of CM319 cells at a density of $1 \times 10^{7}$ were injected subcutaneously into the NOD/SCID mice. Tumor volumes were examined every other day and the volume of the tumor $(\mathrm{V})$ was approximately calculated as $V=(\text { length } \times \text { width })^{2} / 2\left(\mathrm{~mm}^{3}\right)$. When the mean tumor volume reached $500 \mathrm{~mm}^{3}$ ( 15 days after the injection of CM319 cells), the NOD/SCID mice were randomly assigned (using a random number table) to four groups: three mice for each group-control group (Con), siKRT8 group (siKRT8), Doxo-treated group (Doxo, $2 \mathrm{mg} /$ $\mathrm{kg}$ ), and siKRT8 and Doxo-treated group (si + Doxo $2 \mathrm{mg} /$ $\mathrm{kg}$ ). Doxo were intraperitoneally administered every day for 14 days and siRNA was transfected three times weekly ${ }^{59,60}$. The chemically modified stable ${ }^{\mathrm{TM}}$ siRNA of human KRT8 (siKRT8) for in vivo studies was manufactured by Genepharma (Suzhou, China). The stable ${ }^{\mathrm{TM}}$ siRNA $(20 \mu \mathrm{g})$ was dissolved in Optic-MEM medium (Thermo Fisher Scientific, USA, \#31985062) and was injected to the tumor. After 30 days from the injection of the CM319 cells, the mice were killed and xenografts were collected for weighing and histology analysis. The investigator who measured the volume and weight of the tumor was blinded to the group allocation. No animal was excluded from the analysis.

\section{Statistical analysis}

Statistical analysis was performed with SPSS 22.0 and GraphPad Prism 7.0 software. The results were given as mean \pm SD. No statistical methods were used to determine the sample size for in vitro and in vivo experiments. An $F$-test for equality of variances was performed to ensure the same variance of tested groups. The Shapiro- 

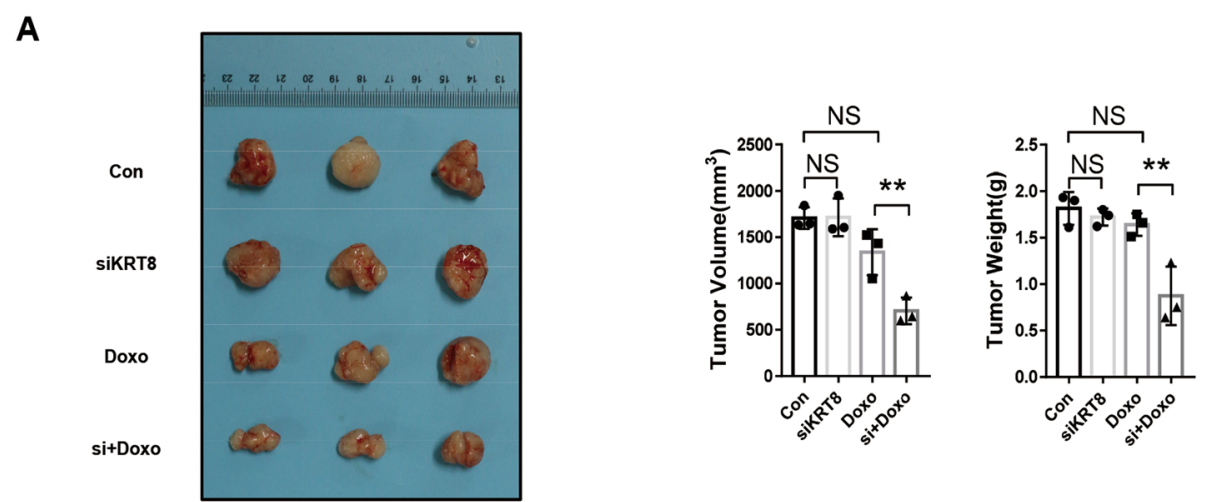

B
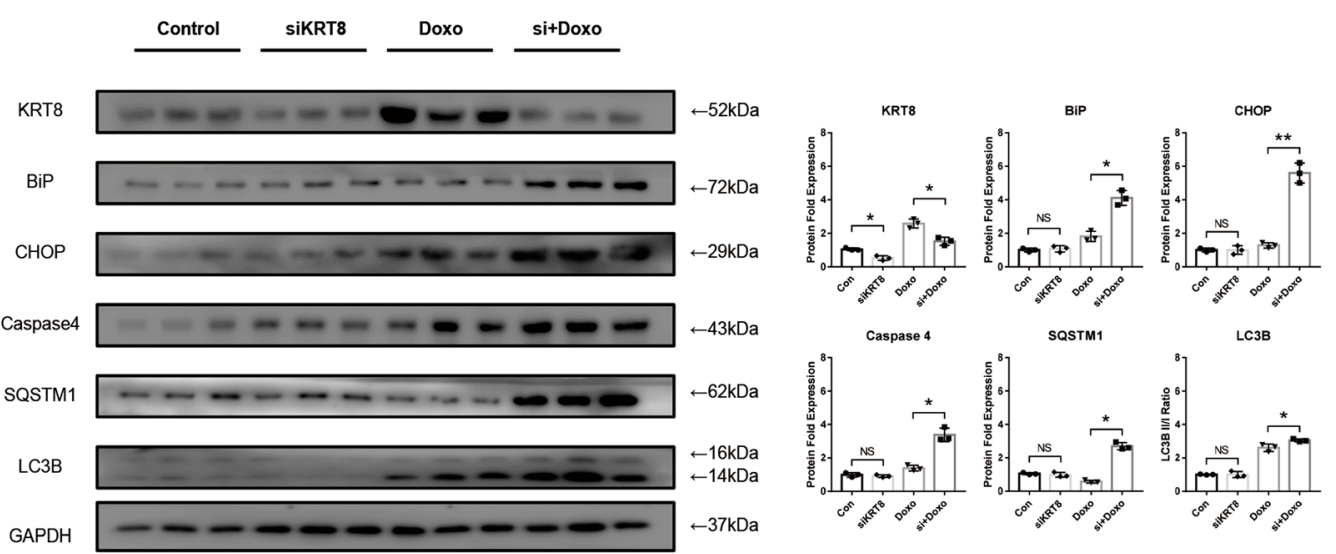

C

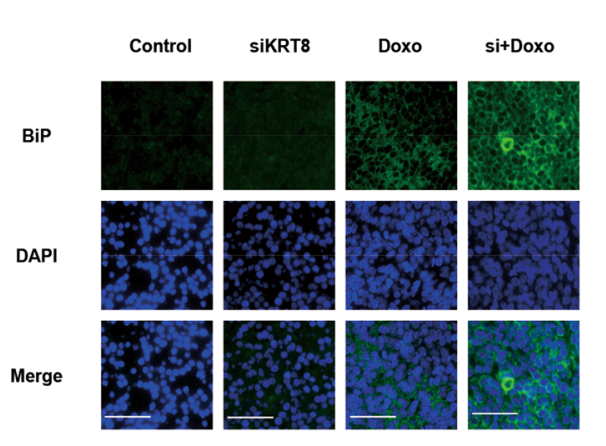

D

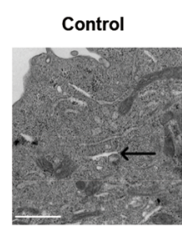

Doxo
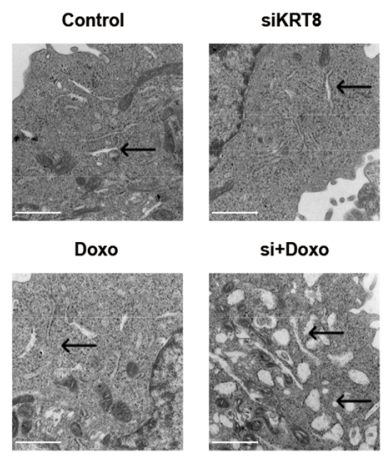

si+Doxo

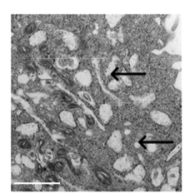

E

Conversion from LC3-I to LC3-II
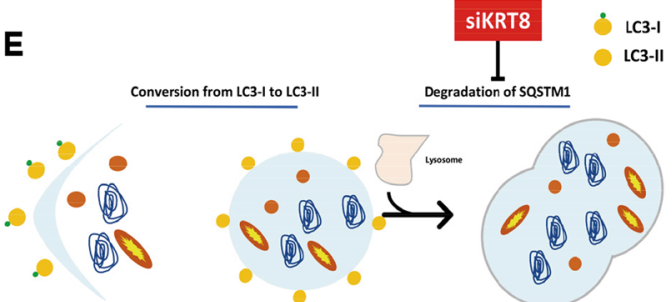

$\uparrow \widehat{\text { KRT8 }} \leftarrow \mathrm{G}_{\text {Chemotherapy }}^{\uparrow}$

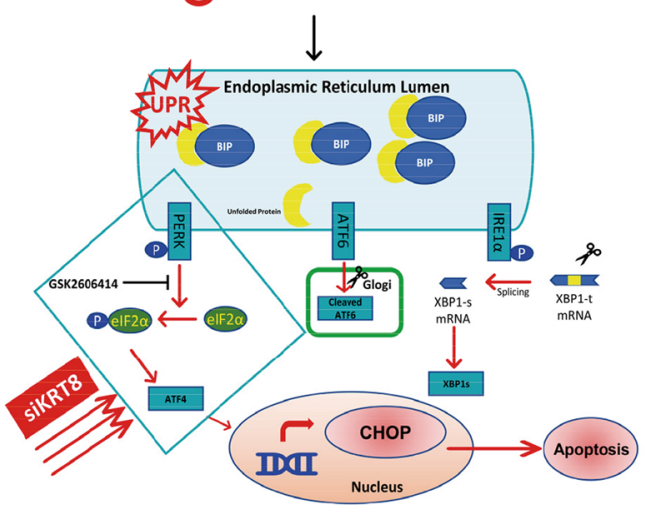

Fig. 8 (See legend on next page.) 
(see figure on previous page)

Fig. 8 Knockdown of KRT8 increased chemosensitivity of chordoma cells in vivo. NOD/SCID mice bearing CM319 cell xenografts were divided into four groups: three mice for each group-(1) control group (Con), (2) siKRT8 group (siKRT8), (3) doxorubicin group (Doxo, 2 mg/kg), and (4) siKRT8 and doxorubicin group (si + Doxo $2 \mathrm{mg} / \mathrm{kg}$ ). a siKRT8 combined with doxorubicin exerted a more significant anti-tumor effect as shown by tumor weights and volume. $\mathbf{b}$ Western blotting analysis and quantification of KRT8, BiP, CHOP, Caspase 4, SQSTM1, and LC3B protein expression of tumor xenograft tissues (normalized to GAPDH expression). c Immunofluorescence staining of BiP of tumor xenograft sections (scale bar $=100 \mu \mathrm{m}$ ). d Representative TEM image of tumor tissues, ER was evidently distended in si + Doxo-treated groups (arrowhead, scale bar $=1 \mu \mathrm{m}$ ). e Proposed mechanism of KRT8 in chordoma drug resistance. KRT8 of chordoma cells is upregulated after chemotherapy, accompanied by increased autophagy activity and ER stress. Knockdown of KRT8 sensitizes chordoma cells to chemotherapy by blocking late-stage autophagy and aggravating ER stress through PERK/elF2a arm of UPR $\left(n=3,{ }^{*} p<0.05\right.$ vs. indicated group, ${ }^{* *} p<0.01$ vs. indicated group, NS: not statistically significant vs. indicated group, Con: control group, Doxo: doxorubicin-treated group. For all the above-mentioned statistical analyses, significance was determined by one-way ANOVA followed by Tukey's multiple comparisons test and the results were shown as mean \pm SD).

Wilk test or D'Agostino test was performed to determine whether the data follow a normal distribution. Differences between experimental groups were assessed using oneway analysis of variance followed by Dunnett's or Tukey's multiple comparisons test. A $p<0.05$ was considered statistically significant.

\section{Acknowledgements}

This work was supported by grants from the National Natural Science Foundation of China (81730065 and 81772377).

\section{Author details}

'Institute of Orthopedic Surgery, Xijing Hospital, Fourth Military Medical University, Xi'an 710032, China. ${ }^{2}$ Department of Biochemistry and Molecular Biology, Fourth Military Medical University, Xi'an 710032, China. ${ }^{3}$ Medical Research Institute, Northwestern Polytechnical University, Xi'an 710032, China. ${ }^{4}$ Wellcome Centre for Cell Matrix Research, Division of Cell Matrix Biology and Regenerative Medicine, School of Biological Sciences, Faculty of Biology, Medicine and Health, Manchester Academic Health Science Centre, University of Manchester, Manchester, UK

\section{Conflict of interest}

The authors declare that they have no conflict of interest.

\section{Publisher's note}

Springer Nature remains neutral with regard to jurisdictional claims in published maps and institutional affiliations.

Supplementary Information accompanies this paper at (https://doi.org/ 10.1038/s41419-019-2125-9).

Received: 1 August 2019 Revised: 23 October 2019 Accepted: 6 November 2019

Published online: 25 November 2019

\section{References}

1. Azzarelli, A. et al. Chordoma: natural history and treatment results in 33 cases. J. Surg. Oncol. 37, 185-191 (1988).

2. Ji, Z. et al. Expression of MDR1, HIF-1alpha and MRP1 in sacral chordoma and chordoma cell line CM-319. J. Exp. Clin. Cancer Res. 29, 158 (2010).

3. Kerr, D. L. et al. Epidemiologic and survival trends in adult primary bone tumors of the spine. Spine J. (2019). https://doi.org/0.1016/j.spinee.2019.07.003. (In press).

4. Colia, V. \& Stacchiotti, S. Medical treatment of advanced chordomas. Eur. J. Cancer 83, 220-228 (2017).

5. van Wulfften, P. O. et al. Sacral chordoma: a clinical review of 101 cases with 30-year experience in a single institution. Spine J. 19, 869-879 (2019).
6. Stacchiotti, S. \& Sommer, J. Building a global consensus approach to chordoma: a position paper from the medical and patient community. Lancet Oncol. 16, e71-e83 (2015).

7. McMaster, M. L., Goldstein, A. M., Bromley, C. M., Ishibe, N. \& Parry, D. M. Chordoma: incidence and survival patterns in the United States, 1973-1995. Cancer Causes Control. 12, 1-11 (2001).

8. Cao, X. et al. Combination of PARP inhibitor and temozolomide to suppress chordoma progression. J. Mol. Med. (Berl). 97, 1183-1193 (2019).

9. Gulluoglu, S. et al. The role of TNF-alpha in chordoma progression and inflammatory pathways. Cell Oncol. (Dordr). 42, 1-15 (2019).

10. Liao, J., Ku, N. O. \& Omary, M. B. Stress, apoptosis, and mitosis induce phosphorylation of human keratin 8 at Ser-73 in tissues and cultured cells. J. Biol. Chem. 272, 17565-17573 (1997).

11. Kelley, M. J. et al. Characterization of T gene sequence variants and germline duplications in familial and sporadic chordoma. Hum. Genet. 133, 1289-1297 (2014).

12. Gulluoglu, S., Turksoy, O., Kuskucu, A., Ture, U. \& Bayrak, O. F. The molecular aspects of chordoma. Neurosurg. Rev. 39, 185-196 (2016). Discussion 196.

13. Presneau, $N$. et al. Role of the transcription factor $T$ (brachyury) in the pathogenesis of sporadic chordoma: a genetic and functional-based study. J. Pathol. 223, 327-335 (2011).

14. Gilbert, S., Loranger, A., Daigle, N. \& Marceau, N. Simple epithelium keratins 8 and 18 provide resistance to Fas-mediated apoptosis. The protection occurs through a receptor-targeting modulation. J. Cell Biol. 154, 763-773 (2001).

15. Ku, N. O. \& Omary, M. B. A disease- and phosphorylation-related nonmechanical function for keratin 8. J. Cell Biol. 174, 115-125 (2006).

16. Baek, A. et al. Autophagy and KRT8/keratin 8 protect degeneration of retinal pigment epithelium under oxidative stress. Autophagy 13, 248-263 (2017).

17. Guldiken, N. et al. Human keratin 8 variants promote mouse acetaminophen hepatotoxicity coupled with c-jun amino-terminal kinase activation and protein adduct formation. Hepatology 62, 876-886 (2015).

18. Asghar, M. N. et al. Keratin 8 knockdown leads to loss of the chloride transporter DRA in the colon. Am. J. Physiol. Gastrointest. Liver Physiol. 310, G1147-G1154 (2016).

19. Lahdeniemi, I. et al. Keratins regulate colonic epithelial cell differentiation through the Notch1 signalling pathway. Cell Death Differ. 24, 984-996 (2017).

20. Vijayaraj, P. et al. Keratins regulate protein biosynthesis through localization of GLUT1 and -3 upstream of AMP kinase and Raptor. J. Cell Biol. 187, 175-184 (2009).

21. Chen, Y. et al. Loss of keratin 19 favours the development of cholestatic liver disease through decreased ductular reaction. J. Pathol. 237, 343-354 (2015).

22. Trisdale, S. K., Schwab, N. M., Hou, X., Davis, J. S. \& Townson, D. H. Molecular manipulation of keratin 8/18 intermediate filaments: modulators of FASmediated death signaling in human ovarian granulosa tumor cells. J. Ovarian Res. 9,8 (2016).

23. Hetz, C. The unfolded protein response: controlling cell fate decisions under ER stress and beyond. Nat. Rev. Mol. Cell Biol. 13, 89-102 (2012).

24. Ron, D. \& Walter, P. Signal integration in the endoplasmic reticulum unfolded protein response. Nat. Rev. Mol. Cell Biol. 8, 519-529 (2007).

25. Zheng, C. et al. Suppressing UPR-dependent overactivation of FGFR3 signaling ameliorates SLC26A2-deficient chondrodysplasias. Ebiomedicine 40, 695-709 (2019). 
26. Liu, Z., LV, Y., Zhao, N., Guan, G. \& Wang, J. Protein kinase R-like ER kinase and its role in endoplasmic reticulum stress-decided cell fate. Cell Death Dis. 6 , e1822 (2015).

27. Avril, T., Vauleon, E. \& Chevet, E. Endoplasmic reticulum stress signaling and chemotherapy resistance in solid cancers. Oncogenesis 6, e373 (2017).

28. Moenner, M., Pluquet, O., Bouchecareilh, M. \& Chevet, E. Integrated endoplasmic reticulum stress responses in cancer. Cancer Res. 67, 10631-10634 (2007).

29. Mann, M. J. \& Hendershot, L. M. UPR activation alters chemosensitivity of tumor cells. Cancer Biol. Ther. 5, 736-740 (2006).

30. Chern, Y. J. et al. The interaction between SPARC and GRP78 interferes with ER stress signaling and potentiates apoptosis via PERKVIF2alpha and IRE1alpha/ XBP-1 in colorectal cancer. Cell Death Dis. 10, 504 (2019).

31. Gao, B. et al. Eicosapentaenoic acid attenuates dexamethasome-induced apoptosis by inducing adaptive autophagy via GPR120 in murine bone marrow-derived mesenchymal stem cells. Cell Death Dis. 7, e2235 (2016).

32. Leidal, A. M., Levine, B. \& Debnath, J. Autophagy and the cell biology of agerelated disease. Nat. Cell Biol. 20, 1338-1348 (2018).

33. Hurley, J. H. \& Young, L. N. Mechanisms of autophagy initiation. Annu Rev. Biochem. 86, 225-244 (2017).

34. Shen, S. et al. Blocking autophagy enhances the apoptotic effect of 18betaglycyrrhetinic acid on human sarcoma cells via endoplasmic reticulum stress and JNK activation. Cell Death Dis. 8, e3055 (2017).

35. Sui, X. et al. Autophagy and chemotherapy resistance: a promising therapeutic target for cancer treatment. Cell Death Dis. 4, e838 (2013).

36. Huang, Z., Zhou, L., Chen, Z., Nice, E. C. \& Huang, C. Stress management by autophagy: Implications for chemoresistance. Int J. cancer 139, 23-32 (2016).

37. Belounis, A. et al. Autophagy is associated with chemoresistance in neuroblastoma. BMC Cancer 16, 891 (2016).

38. Kolb-Lenz, D. et al. Characterization of the endolysosomal system in human chordoma cell lines: is there a role of lysosomes in chemoresistance of this rare bone tumor? Histochem. Cell Biol. 150, 83-92 (2018).

39. Yang, $C$. et al. Characterization and analysis of human chordoma cell lines. Spine (Philos. PA 1976). 35, 1257-1264 (2010).

40. Bruderlein, S. et al. Molecular characterization of putative chordoma cell lines. Sarcoma 2010, 630129 (2010).

41. Vitner, E. B., Platt, F. M. \& Futerman, A. H. Common and uncommon pathogenic cascades in lysosomal storage diseases. J. Biol. Chem. 285, 20423-20427 (2010).

42. Yang, L., Li, P., Fu, S., Calay, E. S. \& Hotamisligil, G. S. Defective hepatic autophagy in obesity promotes ER stress and causes insulin resistance. Cell Metab. 11, 467-478 (2010)

43. Szegezdi, E., Logue, S. E., Gorman, A. M. \& Samali, A. Mediators of endoplasmic reticulum stress-induced apoptosis. EMBO Rep. 7, 880-885 (2006).
44. Senft, D. \& Ronai, Z. A. UPR, autophagy, and mitochondria crosstalk underlies the ER stress response. Trends Biochem Sci. 40, 141-148 (2015).

45. Zhang, $H$. et al. miR-16-5p inhibits chordoma cell proliferation, invasion and metastasis by targeting Smad3. Cell Death Dis. 9, 680 (2018).

46. Sharifnia, T. et al. Small-molecule targeting of brachyury transcription factor addiction in chordoma. Nat. Med. 25, 292-300 (2019).

47. Chugh, R. et al. Phase II study of 9-nitro-camptothecin in patients with advanced chordoma or soft tissue sarcoma. J. Clin. Oncol. 23, 3597-3604 (2005).

48. Pekny, M. \& Lane, E. B. Intermediate filaments and stress. Exp. Cell Res. 313 2244-2254 (2007).

49. Binet, F. \& Sapieha, P. ER stress and angiogenesis. Cell Metab. 22, 560-575 (2015).

50. Mahoney, E. et al. ER stress and autophagy: new discoveries in the mechanism of action and drug resistance of the cyclin-dependent kinase inhibitor flavopiridol. Blood 120, 1262-1273 (2012).

51. Hetz, C., Chevet, E. \& Harding, H. P. Targeting the unfolded protein response in disease. Nat. Rev. Drug Discov. 12, 703-719 (2013).

52. Liao, Z. et al. Exosomes from mesenchymal stem cells modulate endoplasmic reticulum stress to protect against nucleus pulposus cell death and ameliorate intervertebral disc degeneration in vivo. Theranostics 9 4084-4100 (2019).

53. Wang, Z. et al. CYT997(Lexibulin) induces apoptosis and autophagy through the activation of mutually reinforced ER stress and ROS in osteosarcoma. J. Exp. Clin. Cancer Res. 38, 44 (2019).

54. Urra, H., Dufey, E., Lisbona, F., Rojas-Rivera, D. \& Hetz, C. When ER stress reaches a dead end. Biochim. Biophys. Acta 1833, 3507-3517 (2013).

55. Li, Y., Guo, Y., Tang, J., Jiang, J. \& Chen, Z. New insights into the roles of CHOPinduced apoptosis in ER stress. Acta Biochim. Biophys. Sin. (Shanghai) 46, 629-640 (2014).

56. Ogata, M. et al. Autophagy is activated for cell survival after endoplasmic reticulum stress. Mol. Cell Biol. 26, 9220-9231 (2006).

57. Kongara, S. et al. Autophagy regulates keratin 8 homeostasis in mammary epithelial cells and in breast tumors. Mol. Cancer Res. 8, 873-884 (2010)

58. Zhang, D. Z., Ma, B. A., Fan, Q. Y., Chang, H. \& Wen, Y. H. Establishment and characteristics of a human chordoma cell line. Zhonghua Zhong Liu Za Zhi. 25, 234-237 (2003).

59. Pille, J. Y. et al. Anti-RhoA and anti-RhoC siRNAs inhibit the proliferation and invasiveness of MDA-MB-231 breast cancer cells in vitro and in vivo. Mol. Ther. 11, 267-274 (2005)

60. Guan, $H$. et al. A small interfering RNA targeting vascular endothelial growth factor inhibits Ewing's sarcoma growth in a xenograft mouse model. Clin Cancer Res. 11, 2662-2669 (2005). 